\title{
The Properties of Graphs of Matroids
}

\author{
Ping $\mathrm{Li}^{1}$ and Guizhen $\mathrm{Liu}^{2, \dagger}$ \\ ${ }^{1}$ Department of Mathematics, West Virginia University, Morgantown, WV \\ ${ }^{2}$ School of Mathematics and System Science, Shandong University, Jinan \\ 1 USA \\ ${ }^{2}$ China
}

\section{Introduction}

Let $E$ be a finite set of elements. For $S_{1}, S_{2} \subseteq E$, set $S_{1}-S_{2}=\left\{x \mid x \in S_{1}\right.$ and $\left.x \notin S_{2}\right\}$. Let $\mathcal{C}$ be a collection of non-null subsets of $E$ which satisfies the following two axioms.

(C1) A proper subset of a member of $\mathcal{C}$ is not a member of $\mathcal{C}$.

(C2) If $a \in C_{1} \cap C_{2}$ and $b \in C_{1}-C_{2}$ where $C_{1}, C_{2} \in \mathcal{C}$ and $a, b \in E$, then there exists a $C_{3} \in \mathcal{C}$ such that $b \in C_{3} \subseteq\left(C_{1} \cup C_{2}\right)-\{a\}$.

Then $M=(E, \mathcal{C})$ is called a matroid on $E$. We refer to the members of $\mathcal{C}$ as circuits of matroid $M$. The set of bases of a matroid $M$ is a nonempty collection $\mathcal{B}$ of subsets of $E$ such that the following condition is satisfied. For any $B, B^{\prime} \in \mathcal{B},|B|=\left|B^{\prime}\right|$ and for any $e \in B \backslash B^{\prime}$, there exists $e^{\prime} \in B^{\prime} \backslash B$ such that $(B \backslash\{e\}) \cup\left\{e^{\prime}\right\} \in \mathcal{B}$, We also write as $M=(E, \mathcal{B})$. Each member of $\mathcal{B}$ is called a base of $M$. The rank $r$ of a matroid is the number of elements in a base and the co-rank $r^{*}$ is the number of its basic circuits. If $e \in E \backslash B$, then $B \cup\{e\}$ contains a unique basic circuit and denoted by $C(e, B)$. For a given base $B$ of $M$, the set of basic circuits with respect to $B$ is denoted by $\mathcal{C}_{B}$. We use $\mathcal{B}_{e}$ and $\overline{\mathcal{B}}_{e}$ to denote the set of bases containing $e$ and avoiding $e$, respectively. Let $M=(E, \mathcal{C})$ be a matroid. If $X \subseteq E$, then the matroid on $E-X$ whose circuits are those of $M$ which are contained in $E-X$ is called the restriction of $M$ to $E-X$ (or the matroid obtained by deleting $X$ from $M$ ) and is denoted by $M \backslash X$ or $M \mid(E-X)$. There is another derived matroid of importance. If $X \subseteq E$, then the family of minimal non-empty intersections of $E-X$ with circuits of $M$ is the family of circuits of a matroid on $E-X$ called the contraction of $M$ to $E-X$. If $X=\{e\}$, we use $M \backslash e$ and $M / e$ to denote the matroid obtained from $M$ by deleting and contracting $e$, respectively. A matroid obtained from $M$ by limited times of contractions and limited times of deletions is called a minor of $M$. A subset $S$ of $E$ is called a separator of $M$ if every circuit of $M$ is either contained in $S$ or $E-S$. Union and intersection of two separators of $M$ is also a separator of $M$. If $\varnothing$ and $E$ are the only separators of $M$, then $M$ is said to be connected. The minimal non-empty separators of $M$ are called the

*This research was supported by NNSF(61070230) of China.

tCorresponding Author 
components of $M$. The base graph of matroid $M$ is a graph $G_{B}(M)$ with vertex set $V\left(G_{B}\right)$ and edge set $E\left(G_{B}\right)$ such that $V\left(G_{B}\right)=\mathcal{B}$ and $E\left(G_{B}\right)=\left\{B B^{\prime}\left|B, B^{\prime} \in \mathcal{B},\right|\left|B-B^{\prime}\right|=1\right\}$.

Let $G$ be a graph. The vertex set and edge set of a graph $G$ are denoted by $V(G)$ and $E(G)$, respectively. If $A \subseteq V(G)$, then $G[A]$ denotes the induced subgraph of $G$ by $A$. A $k$-path is a path of $k$-edges and denoted by $P_{k}$. A $k$-circuit is a circuit of $k$-edges and denoted by $C_{k}$. $K_{n}$ denotes the complete graph of order $n$. A graph is Hamiltonian connected if for any two vertices there is a Hamilton path connects them. A graph is Hamiltonian if it contains a Hamilton circuit. A graph $G$ is positively Hamiltonian, written $G \in H^{+}$, if for every edge of $G$, there is a Hamilton circuit containing it. $G$ is negatively Hamiltonian, written $G \in H^{-}$, if for every edge of $G$, there is a Hamilton circuit avoiding it. When $G \in H^{+}$and $G \in H^{-}$, we say that $G$ is uniformly Hamiltonian. If for every edge $e$ of $G$, there is a $k$-circuit containing it for any $k, 3 \leq k \leq|V(G)|$, then $G$ is called edge-pancyclic. A graph $G$ is called $E_{2}$-Hamiltonian if every two edges of $G$ are contained in a Hamilton cycle of $G$. Let $G$ be a simple graph of order at least 3 vertices. Then graph $G$ is called $p_{3}$-Hamilton, if for any path $P$ with 3 vertices, there exists a Hamilton cycle of $G$ which contains $P$. If for any two vertices $v_{1}$ and $v_{2}$ and any edge $v_{2} v_{3}$ where $v_{1} \neq v_{3}$, graph $G$ has a Hamilton path from $v_{1}$ to $v_{2}$ and such that edge $v_{2} v_{3}$ in this path, then we say that graph $G$ is $1-H a m i l t o n$ connected. Terminology and notations not defined here can be found in [1] and [2].

Maurer defined the base graph of a matroid, and discussed the graphical properties of the base graph of a matroid [3-4]. Cummins showed that every matroid base graph with at least three vertices has a Hamilton circuit [5]. Holzmann and Harary showed that for every edge in a base graph there is a Hamilton circuit containing it and another Hamilton circuit avoiding it [6]. Alspach and Liu studied the properties of paths and circuits in base graphs of matroids [7]. The connectivity of the base graph of matroids is investigated by Liu [8]. The other graphical properties of the base graphs of matroid have also been investigated by Liu [9-16].

Now we give a new concept as follows. The circuit graph of a matroid $M$ is a graph $G_{C}=$ $G(M)$ with vertex set $V\left(G_{C}\right)$ and edge set $E\left(G_{C}\right)$ such that $V\left(G_{C}\right)=\mathcal{C}$ and $E\left(G_{C}\right)=\left\{C C^{\prime} \mid\right.$ $\left.C, C^{\prime} \in \mathcal{C},\left|C \cap C^{\prime}\right| \neq 0\right\}$, where the same notation is used for the vertices of $G$ and the circuits of $M$. We give another new graph related to the bases of matroids as follows. The intersection graph of bases of matroid $M=(E, \mathcal{B})$ is a graph $G_{I}(M)$ with vertex set $V\left(G_{I}\right)$ and edge set $E\left(G_{I}\right)$ such that $V\left(G_{I}\right)=\mathcal{B}$ and $E\left(G_{I}\right)=\left\{B B^{\prime}:\left|B \cap B^{\prime}\right| \neq 0, B, B^{\prime} \in \mathcal{B}(M)\right\}$, where the same notation is used for the vertex of $G_{I}$ and the base of $M$. The properties of paths, cycles and the connectivity of circuit graphs of matroids are discussed in this chapter. In particular, some new results obtained by us are given.

\section{Preliminary results}

To prove the main theorem we need the following preliminary results.

Lemma 2.1. [17] A matroid $M$ is connected if and only if for every pair $e_{1}, e_{2}$ of distinct elements of $E$, there is a circuit containing both $e_{1}$ and $e_{2}$.

Lemma 2.2. [17] If $M$ is a connected matroid, then for every $e \in E$, either $M / e$ or $M \backslash e$ is also connected. 
Lemma 2.3. [17] Let $C$ and $C^{\star}$ be any circuit and co-circuit of a matroid $M$. Then $\left|C \cap C^{\star}\right| \neq 1$.

Lemma 2.4. [1] If $a \in C_{1} \cap C_{2}$ and $b \in C_{1}-C_{2}$ where $C_{1}, C_{2} \in \mathcal{C}$, then there exists a $C_{3} \in \mathcal{C}$ such that $b \in C_{3} \subseteq\left(C_{1} \cup C_{2}\right)-\{a\}$.

Let $M=(E, \mathcal{C})$ be a connected matroid. An element $e$ of $E$ is called an essential element if $M \backslash e$ is disconnected. Otherwise it is called an inessential element. A connected matroid each of whose elements is essential is called a critically connected matroid or simply a critical matroid.

Lemma 2.5. [17] A critical matroid of rank 2 contains a co-circuit of cardinality two.

A matroid $M$ is trivial if it has no circuits. In the following matroids will be nontrivial. Next we will discuss the properties of the matroid circuit graph. To prove the main results we firstly present the following result which is clearly true.

Lemma 2.6. [17] Let $M$ be any nontrivial matroid on $E$ and $e \in E$. If $G_{C}$ and $G_{C e}$ are circuit graphs of $M$ and $M \backslash e$, respectively, then $G_{C e}$ is a subgraph of $G$ induced by $V_{1}$ where $V_{1}=$ $\{C \mid C \in \mathcal{C}, e \notin C\}$.

Obviously the subgraph $G_{C 2}$ of $G$ induced by $V_{2}=V-V_{1}$ is a complete graph. By Lemma 2.6 $G_{C 1}$ and $G_{C 2}$ are induced subgraphs of $G$ and $V\left(G_{C 1}\right)$ and $V\left(G_{C 2}\right)$ partition $V(G)$.

Lemma 2.7. [17] For any matroid $M=(E, \mathcal{C})$ which has a 2-cocircuit $\{a, b\}$, the circuit graph of $M$ is isomorphic to that of $M / a$.

Proof. Since $|C \cap\{a, b\}| \neq 1$ for any circuit $C$, by Lemma 2.3, the circuits of $M$ can be partitioned into two classes, those circuits containing both $a$ and $b$ and those circuits containing neither $a$ nor $b$. Likewise, the circuits of $M / a$ can be partitioned into two classes: those containing $b$ and those not containing $b$, clearly there is a bijection between $\mathscr{C}(M)$ and $\mathscr{C}(M / a)$. Hence $G(M) \cong G(M / a)$, the lemma is proved.

Lemma 2.8. [17] Suppose that $M=(E, \mathcal{C})$ is a connected matroid with an element $e$ such that the matroid $M \backslash e$ is connected and $G=G_{C}(M)$ is the circuit graph of matroid $M$. Let $G_{1}=G(M \backslash e)$ be the circuit graph of $M \backslash e$ and $G_{2}$ be the subgraph of $G$ induced by $V_{2}$ where $V_{2}=\{C \mid C \in \mathcal{C}, e \in C\}$. If the matroid $M \backslash e$ has more than one circuit, then for any edge $C_{1} C_{2} \in E(G)$, there exists a 4-cycle $C_{1} C_{2} C_{3} C_{4}$ in graph $G$ such that one edge of the 4-cycle belongs to $E\left(G_{1}\right)$ and one belongs to $E\left(G_{2}\right)$ and $C_{1}, C_{2}$ are both adjacent to $C_{3}$.

Proof. By Lemma 2.6, $V\left(G_{1}\right)$ and $V\left(G_{2}\right)$ partition $V(G)$. There are three cases to distinguish.

Case 1. $e \in E-\left(C_{1} \cup C_{2}\right)$. Thus $C_{1} C_{2}$ is an edge of $M \backslash e$. By Lemma 2.1, there are at least three vertices in $G(M \backslash e)$. There is an element $e_{1}$ such that $e_{1} \in C_{1} \cap C_{2}$. Let $G_{1}$ and $G_{2}$ be the graphs defined as above. Note that $G_{2}$ is a complete graph. By Lemma 2.1, there is a vertex $C_{3}$ in $G_{2}$ containing both $e_{1}$ and $e$. Thus in $G, C_{3}$ is adjacent to both $C_{1}$ and $C_{2}$. Since $C_{1} \not \subset C_{3}$, there exists $e_{2}$ such that $e_{2} \in C_{1}$, but $e_{2} \notin C_{3}$. By Lemma 2.1, there is a circuit $C_{4}$ in $G_{2}$ containing $e_{2}$ and $e$ and $C_{3} \neq C_{4}$. Thus $C_{4}$ is adjacent to $C_{1}$.

Case 2. $e \in C_{1}-C_{2}$ or $e \in C_{2}-C_{1}$. Suppose that $e \in C_{2}-C_{1}, e_{1} \in C_{1} \cap C_{2}$. By Lemma 2.4, there is a circuit $C_{3} \subseteq\left(C_{1} \cup C_{2}\right)-\left\{e_{1}\right\}$ containing $e$. We assume that $e_{2} \in C_{1} \cap C_{3}$, $e_{3} \in E-\left(C_{1} \cup\{e\}\right)$. Note that $e_{3}$ exists because, by hypothesis, $M \backslash e$ has more than one 
circuit. By Lemma 2.1, in $G_{1}=G(M \backslash e)$ there is a circuit $C_{4}$ containing $e_{2}$ and $e_{3} . C_{1} C_{2} C_{3} C_{4}$ is the 4-cycle we wanted. $C_{1}$ and $C_{2}$ are both adjacent to $C_{3}$.

Case 3. $e \in C_{1} \cap C_{2}$. $C_{1}$ and $C_{2}$ are both in $G_{2}$. If there are only two circuits containing $e$, it is easy to see that $C_{1} \cup C_{2}=E(M)$ by Lemma 2.6. We prove that $C_{1} \cap C_{2}=\{e\}$. If $C_{1} \cap C_{2}=\left\{e, e^{\prime}\right\}$, then $\left\{e, e^{\prime}\right\}$ is a co-circuit of $M$ because by Lemma 2.4, if there is a circuit containing $e^{\prime}$ does not contain $e$, then there is a circuit containing $e$ does not contain $e^{\prime}$; which is a contradiction to the hypothesis. Thus $C_{1} \cap C_{2}=\{e\}$. Then there is only one circuit $C_{3}^{\prime}=\left(C_{1} \cup C_{2}\right)-\{e\}$ in $M \backslash e$. For if there is a circuit $C_{4}^{\prime} \neq C_{3}^{\prime}$ in $M \backslash e$, then there exists $e_{1} \in E(M)-\left(\{e\} \cup C_{4}^{\prime}\right)$ and $e_{1} \in C_{1}-C_{2}$ or $e_{1} \in C_{2}-C_{1}$. We assume that $e_{1} \in C_{1}-C_{2}$. By Lemma 2.4, there is a circuit $C_{5}$ such that $e \in C_{5} \subseteq\left(C_{2} \cup C_{4}^{\prime}\right)-\left\{e_{2}\right\}$ where $e_{2} \in C_{2} \cap C_{4}^{\prime}$. $e_{1} \notin C_{5}, C_{5} \neq C_{1}$, thus there are at least three circuits containing $e$, a contradiction. So there are more than two circuits containing $e$, we assume that $e_{3} \in C_{1}-C_{2}$. By Lemma 2.4, there is $C_{3}$ in $G_{1}$ such that $e_{3} \in C_{3} \subseteq\left(C_{1} \cup C_{2}\right)-\{e\}$. By Lemma 2.1, there is a vertex $C_{4}$ in $G_{1}$ containing $e_{3}$ and $e_{4}$ where $e_{4} \in E-\left(C_{3} \cup\{e\}\right)$. We get the 4-cycle $C_{1} C_{2} C_{3} C_{4}$. The proof is completed.

Lemma 2.9. [17] Suppose that $M=(E, \mathcal{C})$ is a connected matroid with an element $e$ such that the matroid $M \backslash e$ is connected and $G=G(M)$ is the circuit graph of matroid $M$. Let $G_{1}=G(M \backslash e)$ be the circuit graph of $M \backslash e$ and $G_{2}$ be the subgraph of $G$ induced by $V_{2}$ where $V_{2}=\{C \mid C \in \mathcal{C}, e \in C\}$. If $C_{1} \in V\left(G_{1}\right), C_{2} \in V\left(G_{2}\right)$ and $d\left(C_{1}, C_{2}\right)=2$, there exists a 3-path $C_{1} C_{3} C_{4} C_{2}$ in graph $G$ such that $C_{3} \in V\left(G_{1}\right), C_{4} \in V\left(G_{2}\right)$ and $C_{1}, C_{2}$ are both adjacent to $C_{3}$ and $C_{4}$.

Proof. By Lemma 2.6, $V\left(G_{1}\right)$ and $V\left(G_{2}\right)$ partition $V(G)$. By assumption, $\left|C_{1} \cap C_{2}\right|=0$. We assume that $e_{1} \in C_{1}, e_{2} \in C_{2}$. By Lemma 2.1, there is $C_{4}$ in $G_{2}$ containing both $e_{1}$ and $e$. Thus in $G, C_{4}$ is adjacent to $C_{1}$ and $C_{2}$. By Lemma 2.1, there is $C_{3}$ in $G_{1}$ containing both $e_{1}$ and $e_{2}$. Thus in $G, C_{3}$ is adjacent to $C_{1}, C_{2}$ and $C_{4}$

Let $P_{4}$ be the Hamilton path connecting $C_{1}$ and $C_{2}$ in $G(M \backslash e)$ which traverses $C_{1}^{\prime} C_{2}^{\prime}$ and $P_{2}$ be the $m$-path connecting $C_{3}$ and $C_{4}$ in $G_{2}\left(1 \leq m \leq n_{2}-1\right)$, respectively. $P_{3}+C_{1}^{\prime} C_{3}+C_{3} C_{2}^{\prime}+P_{4}$ is a $n_{1}$-path of $G(M)$ that joins $C_{1}$ and $C_{2} . P_{3}+C_{1}^{\prime} C_{4}+P_{2}+C_{3} C_{2}^{\prime}+P_{4}$ is a $n_{1}+m$-path of $G(M)$ that joins $C_{1}$ and $C_{2}$.

Subcase 1.2. $e \in C_{1}-C_{2}$ or $e \in C_{2}-C_{1}$. Suppose that $e \in C_{2}-C_{1}$. Thus $C_{1} \in V\left(G_{1}\right), C_{2} \in$ $V\left(G_{2}\right)$. If $d\left(C_{1}, C_{2}\right)=1$, by Lemma 2.8, there is a 4-cycle $C_{1} C_{2} C_{3} C_{4}$ in $G$ such that $C_{3} \in V\left(G_{2}\right)$ and $C_{4} \in V\left(G_{1}\right)$ and $C_{1} C_{2} C_{3}$ is a 3-cycle of $G$. By induction, for any $k_{1}, 1 \leq k_{1} \leq n_{1}-1$, there is a $k_{1}$-path in $G_{1}$ connecting $C_{4}$ and $C_{1}$. Note that $G_{2}$ is a complete graph. For any $k_{2}, 1 \leq k_{2} \leq$ $n_{2}-1$, there is a $k_{2}$-path in $G_{2}$ connecting $C_{2}$ and $C_{3}$. Let $P_{1}$ be the $k_{1}$-path in $G_{1}$ connecting $C_{1}$ and $C_{4}$ and $P_{2}$ be the $k_{2}$-path in $G_{2}$ connecting $C_{3}$ and $C_{2}$, respectively. $P_{1}+C_{4} C_{3}+P_{2}$ is a $k_{1}+k_{2}+1$-path of $G(M)$ that connects $C_{1}$ and $C_{2}$. If $d\left(C_{1}, C_{2}\right)=2$ and $e \in C_{2}-C_{1}$, by Lemma 2.9, there is a 3-path $C_{1} C_{3} C_{4} C_{2}$ in $G$ such that $C_{3} \in V\left(G_{1}\right)$ and $C_{4} \in V\left(G_{2}\right)$ and $C_{1} C_{3} C_{2}$ is a 2-path of $G$. Then the proof is similar to the case when $d\left(C_{1}, C_{2}\right)=1$.

Subcase 1.3. $e \in C_{1} \cap C_{2}$. Thus $d\left(C_{1}, C_{2}\right)=1$. If there are only two circuits containing $e$, then there is only one circuit in $M \backslash e$. The result holds obviously because $G=K_{3}$. Assume that there are more than two circuits containing $e$. Note that $G_{2}$ is a complete graph. For any $m, 1 \leq m \leq n_{2}-1$, there is a path of length $m$ connecting $C_{1}$ and $C_{2}$. Choose $C_{3} \in V\left(G_{2}\right)$ 
such that $C_{3} \neq C_{1}$ and $C_{3} \neq C_{2}$. By Lemma 2.8, there is a 4-cycle $C_{1} C_{3} C_{4} C_{5}$ in $G$ such that $C_{4} C_{5} \in E\left(G_{1}\right)$ and $C_{1} C_{3} C_{4}$ is a 3-cycle of $G$. By induction, for any $k, 1 \leq k \leq n_{1}-1$, there is a $k$-path in $G_{1}$ connecting $C_{4}$ and $C_{5}$. Note that $G_{2}$ is a complete graph. Let $P_{1}$ be the $k$-path in $G_{1}$ connecting $C_{4}$ and $C_{5}$ and $P_{2}$ be the Hamilton path in $G_{2}$ connecting $C_{1}$ and $C_{2}$ which traverses the edge $C_{1} C_{3}$. Let $P_{2}=C_{1} C_{3}+P_{3} . C_{1} C_{4}+C_{4} C_{3}+P_{3}$ is a $n_{2}$-path that connects $C_{1}$ and $C_{2} \cdot C_{1} C_{5}+P_{1}+C_{4} C_{3}+P_{3}$ is the $n_{2}+k$-path we wanted.

Case 2. The matroid $M$ is critically connected. By Lemma 2.2 , for any element $e$ in $M, M / e$ is connected. By Lemma 2.5, $M$ has a 2-cocircuit $\{a, b\}$. By Lemma 2.7, the circuit graph of $M / a$ is isomorphic to that of $M$. By induction hypothesis, the result holds.

Thus the theorem follows by induction.

Lemma 2.10. [2] A graph $G$ is $k$-edge-connected if and only if any two distinct vertices of $G$ are connected by at least $k$ edge- disjoint paths.

\section{The properties of circuit graphs of matroids}

We have known that a matroid $M$ is connected if and only if for every pair $e_{1}, e_{2}$ of distinct elements of $E$, there is a circuit containing both $e_{1}$ and $e_{2}$. The following results are the new results obtained by Li and Liu.

Theorem 3.1. [17] For any connected matroid $M=(E, \mathcal{C})$ which has at least three circuits, the circuit graph $G=G(M)$ is positively Hamiltonian, that is, for every edge of $G$, there is a Hamilton circuit containing it.

We shall prove the theorem by induction on $|E|$. When $|E|=3$, each element in $M$ is parallel to another. It is easy to see that $G=K_{3}$. The theorem is clearly true. Suppose that the result is true for $|E|=n-1$. We prove that the result is also true for $|E|=n>3$. Let $C_{1} C_{2}$ be any edge in $G$.

There are two cases to distinguish.

Case 1. There is an element $e$ in $M$ such that $M \backslash e$ is connected. Let $G_{1}$ and $G_{2}$ be the graphs defined as above. We assume that $\left|V\left(G_{1}\right)\right|=n_{1}$ and $\left|V\left(G_{2}\right)\right|=n_{2}$.

There are three subcases to distinguish.

Subcase 1.1. $e \in E-\left(C_{1} \cup C_{2}\right)$. Thus $C_{1} C_{2}$ is an edge of $M \backslash e$. By Lemma 2.4, there are at least three vertices in $G(M \backslash e)$. By induction, $G(M \backslash e)$ is edge-pancyclic. For any $m, 3 \leq m \leq n_{1}$, there is a cycle of length $m$ containing $C_{1} C_{2}$. By Lemma 2.8 , for any edge $C_{1}^{\prime} C_{2}^{\prime}$ in the Hamilton cycle of $G(M \backslash e)$ containing $C_{1} C_{2}$ where $C_{1}^{\prime} C_{2}^{\prime} \neq C_{1} C_{2}$, there is a 4-cycle $C_{1}^{\prime} C_{2}^{\prime} C_{3} C_{4}$ in $G$ such that $C_{3} C_{4} \in E\left(G_{2}\right)$ and $C_{1}^{\prime} C_{2}^{\prime} C_{3}$ is a 3-cycle in $G$. If there are only three vertices in $G(M \backslash e)$, let $C_{1}=C_{1}^{\prime}$. Note that $G_{2}$ is a complete graph. Let $P_{1}$ be the Hamilton path connecting $C_{1}^{\prime}$ and $C_{2}^{\prime}$ in $G(M \backslash e)$ which traverses $C_{1} C_{2}$ and $P_{2}$ be the $k$-path connecting $C_{3}$ and $C_{4}$ in $G_{2}$ $\left(1 \leq k \leq n_{2}-1\right)$, respectively. $P_{1}+C_{2}^{\prime} C_{3}+C_{3} C_{1}^{\prime}$ is a $n_{1}+1$-cycle of $G(M)$ that contains $C_{1} C_{2}$. $P_{1}+C_{2}^{\prime} C_{3}+P_{2}+C_{4} C_{1}^{\prime}$ is a $n_{1}+k+1$-cycle of $G(M)$ that contains $C_{1} C_{2}$.

Subcase 1.2. $e \in C_{1}-C_{2}$ or $e \in C_{2}-C_{1}$. Suppose that $e \in C_{2}-C_{1}$. Thus $C_{1} \in V\left(G_{1}\right), C_{2} \in$ $V\left(G_{2}\right)$. By Lemma 2.8, there is a 4-cycle $C_{1} C_{2} C_{3} C_{4}$ in $G$ such that $C_{3} \in V\left(G_{2}\right)$ and $C_{4} \in V\left(G_{1}\right)$ and $C_{1} C_{2} C_{3}$ is a 3-cycle of $G$. By induction, for any $k_{1}, 1 \leq k_{1} \leq n_{1}-1$, there is a $k_{1}$-path 
in $G_{1}$ connecting $C_{4}$ and $C_{1}$. Note that $G_{2}$ is a complete graph. For any $k_{2}, 1 \leq k_{2} \leq n_{2}-1$, there is a $k_{2}$-path in $G_{2}$ connecting $C_{2}$ and $C_{3}$. Let $P_{1}$ be the $k_{1}$-path in $G_{1}$ connecting $C_{1}$ and $C_{4}$ and $P_{2}$ be the $k_{2}$-path in $G_{2}$ connecting $C_{3}$ and $C_{2}$, respectively. $P_{1}+C_{4} C_{3}+P_{2}+C_{2} C_{1}$ is a $k_{1}+k_{2}+2$-cycle of $G(M)$ that contains $C_{1} C_{2}$.

Subcase 1.3. $e \in C_{1} \cap C_{2}$. If there are only two circuits containing $e$, then there is only one circuit in $M \backslash e$. The result holds obviously because $G=K_{3}$. Assume that there are more than two circuits containing $e$. Note that $G_{2}$ is a complete graph. For any $m, 3 \leq m \leq n_{2}$, there is a cycle of length $m$ containing $C_{1} C_{2}$. Choose $C_{3} \in V\left(G_{2}\right)$ such that $C_{3} \neq C_{1}$ and $C_{3} \neq C_{2}$. By Lemma 2.8, there is a 4-cycle $C_{1} C_{3} C_{4} C_{5}$ in $G$ such that $C_{4} C_{5} \in E\left(G_{1}\right)$ and $C_{1} C_{3} C_{4}$ is a 3-cycle of $G$. By induction, for any $k, 1 \leq k \leq n_{1}-1$, there is a $k$-path in $G_{1}$ connecting $C_{4}$ and $C_{5}$. Note that $G_{2}$ is a complete graph. Let $P_{1}$ be the $k$-path in $G_{1}$ connecting $C_{4}$ and $C_{5}$ and $P_{2}$ be the Hamilton path in $G_{2}$ connecting $C_{3}$ and $C_{1}$ which traverses the edge $C_{2} C_{1} \cdot C_{4} C_{3}+P_{2}+C_{1} C_{4}$ is a $n_{2}+1$-cycle that contains $C_{1} C_{2} . P_{1}+C_{4} C_{3}+P_{2}+C_{1} C_{5}$ is the $n_{2}+k+1$-cycle we wanted.

Case 2. The matroid $M$ is critically connected. By Lemma 2.2, for any element $e$ in $M, M / e$ is connected. By Lemma 2.5, $M$ has a 2-cocircuit $\{a, b\}$. By Lemma 2.7, the circuit graph of $M / a$ is isomorphic to that of $M$. By induction hypothesis, the result holds.

Thus the theorem follows by induction.

Theorem 3.2. [21] Let $M$ be any connected matroid which has at least four circuits and let $G=G(M)$ be the circuit graph of $M$. Then for each edge of $G=G(M)$ there is a Hamilton cycle avoiding it. that is, the circuit graph $G=G(M)$ is negatively Hamiltonian.

Proof. We prove the theorem by induction on $|E|$. It is easy to see that $|E| \geqslant 4$. When $|E|=4$ and $r(M)=1, M=U_{1,4}[1]$. It is easy to see that $G(M) \in H^{-}$. When $|E|=4$ and $r(M)=2$, $M$ has at most three circuits except when $M=U_{2,4}$. Obviously $G\left(U_{2,4}\right)=K_{4}$ and $K_{4} \in H^{-}$. Then suppose that the theorem is true for $|E|=n-1$. We shall prove the theorem holds for $|E|=n>4$. Let $C_{1} C_{2}$ be any edge of $G$. There are two cases to consider.

Case 1. There exists an element $e$ such that $M \backslash e$ is connected. Let $G_{1}$ and $G_{2}$ be the graphs defined as above.

There are three subcases to consider.

Subcase 1.1. If $e \in E-\left(C_{1} \cup C_{2}\right)$, then $C_{1} C_{2}$ is an edge of $G(M \backslash e)$. By Theorem 3.1, there is a Hamilton cycle in $G(M \backslash e)$ containing $C_{1} C_{2}$. By the proof of Lemma 2.8, there is a 4-cycle $C_{1} C_{2} C_{3} C_{4}$ in $G$ such that $C_{3} C_{4} \in E\left(G_{2}\right)$. As in the proof of Theorem 3.1, let $P_{1}$ be a Hamilton path in $G_{1}$ connecting $C_{1}$ and $C_{2}$ and $P_{2}$ be a Hamilton path in $G_{2}$ connecting $C_{3}$ and $C_{4}$, respectively. Then the cycle $P_{1}+C_{2} C_{3}+P_{2}+C_{4} C_{1}$ is a Hamilton cycle of $G$ avoiding $C_{1} C_{2}$.

Subcase 1.2. Let $e \in C_{1}-C_{2}$ or $e \in C_{2}-C_{1}$. It is easy to see that there are more than two vertices in $G(M \backslash e)$, and there are at least three vertices in $G_{2}$. We assume that $e \in C_{2}-C_{1}$ and $e_{1} \in C_{1}-C_{2}$. By Lemma 2.1, there is $C_{3}$ in $G_{2}$ containing $e$ and $e_{1}$. By the proof of Lemma 2.8, in $G$ there is a 4-cycle $C_{1} C_{3} C_{4} C_{5}$ such that $C_{4} \in V\left(G_{2}\right), C_{5} \in V\left(G_{1}\right)$. Let $P_{1}$ be a Hamilton path in $G(M \backslash e)$ connecting $C_{1}$ and $C_{5}$ and $P_{2}$ be a Hamilton path in $G_{2}$ connecting $C_{4}$ and $C_{3}$, respectively. $P_{1}+C_{5} C_{4}+P_{2}+C_{3} C_{1}$ is a Hamilton cycle avoiding $C_{1} C_{2}$. 
Subcase 1.3. If $e \in C_{1} \cap C_{2}$, as in the proof of subcase 1.3 in Theorem 3.1, let $C_{2}=C_{3}$ and $P_{2}$ be a Hamilton path connecting $C_{1}$ and $C_{2}$ in $G_{2}$, then $P_{1}+C_{4} C_{2}+P_{2}+C_{1} C_{5}$ is a Hamilton cycle we wanted.

Case 2. For every $e \in E(M), M \backslash e$ is disconnected. Then the matroid $M$ is critically connected. By Lemma 2.2, for any element $e$ in $M, M / e$ is connected. By Lemma 2.5, $M$ has a cocircuit $\{a, b\}$. By Lemma 2.7, $G(M) \cong G(M / a)$. By induction hypothesis, the result holds.

The proof of the theorem is completed.

Corollary 3.3. For any connected matroid $M$, the circuit graph $G_{C}(M)$ is uniformly Hamilton whenever $G_{C}(M)$ contains at least four vertices. That is for any edge $e$ of $G_{C}(M)$, there is a Hamilton cycle containing $e$ and there is another Hamilton cycle excluding $e$.

Theorem 3.4. [20] Let $G=G(M)$ be the circuit graph of a connected matroid $M=(E, \mathcal{C})$. If $|V(G)|=n$ and $C_{1}, C_{2} \in V(G)$ with $d\left(C_{1}, C_{2}\right)=r$, then there is a path of length $k$ joining $C_{1}$ and $C_{2}$ for any $k$ satisfying $r \leq k \leq n-1$.

Proof. We shall prove the theorem by induction on $|E|$. When $|E|=3$, each element in $M$ is parallel to another. It is easy to see that $G=K_{3}$. The theorem is clearly true. Suppose that the result is true for $|E|=n-1$. We prove that the result is also true for $|E|=n>3$. It is easy to see that for any vertices $C_{1}, C_{2}$ in $V(G)$, we have $d\left(C_{1}, C_{2}\right)=r$ where $r=1$ or $r=2$ by the definition of the circuit graph of a matroid.

There are two cases to distinguish.

Case 1. There is an element $e$ in $M$ such that $M \backslash e$ is connected. Let $G_{1}$ and $G_{2}$ be the graphs defined as above. We assume that $\left|V\left(G_{1}\right)\right|=n_{1}$ and $\left|V\left(G_{2}\right)\right|=n_{2}$.

There are three subcases to distinguish.

Subcase 1.1. $e \in E-\left(C_{1} \cup C_{2}\right)$. Thus $C_{1} \in V\left(G_{1}\right), C_{2} \in V\left(G_{1}\right)$. Clearly, there are at least three vertices in $G(M \backslash e)$. By induction, for any $k, r \leq k \leq n_{1}-1$, there is a path of length $k$ joining $C_{1}$ and $C_{2}$ in $G(M \backslash e)$. By Lemma 2.8, for any edge $C_{1}^{\prime} C_{2}^{\prime}$ in the Hamilton path connecting $C_{1}$ and $C_{2}$ in $G(M \backslash e)$, there is a 4-cycle $C_{1}^{\prime} C_{2}^{\prime} C_{3} C_{4}$ in $G$ such that $C_{3} C_{4} \in E\left(G_{2}\right)$ and $C_{1}^{\prime} C_{2}^{\prime} C_{3}$ is a 3-cycle in $G$. If there are only three vertices in $G(M \backslash e)$, let $C_{1}=C_{1}^{\prime}$. Note that $G_{2}$ is a complete graph. Let $P_{1}=P_{3}+C_{1}^{\prime} C_{2}^{\prime}+P_{4}$ be the Hamilton path connecting $C_{1}$ and $C_{2}$ in $G(M \backslash e)$ which traverses $C_{1}^{\prime} C_{2}^{\prime}$ and $P_{2}$ be the $m$-path connecting $C_{3}$ and $C_{4}$ in $G_{2}\left(1 \leq m \leq n_{2}-1\right)$, respectively. $P_{3}+C_{1}^{\prime} C_{3}+C_{3} C_{2}^{\prime}+P_{4}$ is a $n_{1}$-path of $G(M)$ that joins $C_{1}$ and $C_{2} \cdot P_{3}+C_{1}^{\prime} C_{4}+P_{2}+C_{3} C_{2}^{\prime}+P_{4}$ is a $n_{1}+m$-path of $G(M)$ that joins $C_{1}$ and $C_{2}$.

Subcase 1.2. $e \in C_{1}-C_{2}$ or $e \in C_{2}-C_{1}$. Suppose that $e \in C_{2}-C_{1}$. Thus $C_{1} \in V\left(G_{1}\right), C_{2} \in$ $V\left(G_{2}\right)$. If $d\left(C_{1}, C_{2}\right)=1$, by Lemma 2.8, there is a 4-cycle $C_{1} C_{2} C_{3} C_{4}$ in $G$ such that $C_{3} \in V\left(G_{2}\right)$ and $C_{4} \in V\left(G_{1}\right)$ and $C_{1} C_{2} C_{3}$ is a 3-cycle of $G$. By induction, for any $k_{1}, 1 \leq k_{1} \leq n_{1}-1$, there is a $k_{1}$-path in $G_{1}$ connecting $C_{4}$ and $C_{1}$. Note that $G_{2}$ is a complete graph. For any $k_{2}, 1 \leq k_{2} \leq n_{2}-1$, there is a $k_{2}$-path in $G_{2}$ connecting $C_{2}$ and $C_{3}$. Let $P_{1}$ be the $k_{1}$-path in $G_{1}$ connecting $C_{1}$ and $C_{4}$ and $P_{2}$ be the $k_{2}$-path in $G_{2}$ connecting $C_{3}$ and $C_{2}$, respectively. $P_{1}+C_{4} C_{3}+P_{2}$ is a $k_{1}+k_{2}+1$-path of $G(M)$ that connects $C_{1}$ and $C_{2}$. 
If $d\left(C_{1}, C_{2}\right)=2$ and $e \in C_{2}-C_{1}$, by Lemma 2.9, there is a 3-path $C_{1} C_{3} C_{4} C_{2}$ in $G$ such that $C_{3} \in V\left(G_{1}\right)$ and $C_{4} \in V\left(G_{2}\right)$ and $C_{1} C_{3} C_{2}$ is a 2-path of $G$. Then the proof is similar to the case when $d\left(C_{1}, C_{2}\right)=1$.

Subcase 1.3. $e \in C_{1} \cap C_{2}$. Thus $d\left(C_{1}, C_{2}\right)=1$. If there are only two circuits containing $e$, then there is only one circuit in $M \backslash e$. The result holds obviously because $G=K_{3}$. Assume that there are more than two circuits containing $e$. Note that $G_{2}$ is a complete graph. For any $m, 1 \leq m \leq n_{2}-1$, there is a path of length $m$ connecting $C_{1}$ and $C_{2}$. Choose $C_{3} \in V\left(G_{2}\right)$ such that $C_{3} \neq C_{1}$ and $C_{3} \neq C_{2}$. By Lemma 2.8, there is a 4-cycle $C_{1} C_{3} C_{4} C_{5}$ in $G$ such that $C_{4} C_{5} \in E\left(G_{1}\right)$ and $C_{1} C_{3} C_{4}$ is a 3-cycle of $G$. By induction, for any $k, 1 \leq k \leq n_{1}-1$, there is a $k$-path in $G_{1}$ connecting $C_{4}$ and $C_{5}$. Note that $G_{2}$ is a complete graph. Let $P_{1}$ be the $k$-path in $G_{1}$ connecting $C_{4}$ and $C_{5}$ and $P_{2}$ be the Hamilton path in $G_{2}$ connecting $C_{1}$ and $C_{2}$ which traverses the edge $C_{1} C_{3}$. Let $P_{2}=C_{1} C_{3}+P_{3} . C_{1} C_{4}+C_{4} C_{3}+P_{3}$ is a $n_{2}$-path that connects $C_{1}$ and $C_{2} \cdot C_{1} C_{5}+P_{1}+C_{4} C_{3}+P_{3}$ is the $n_{2}+k$-path we wanted.

Case 2. The matroid $M$ is critically connected. By Lemma 2.2, for any element $e$ in $M, M / e$ is connected. By Lemma 2.5, $M$ has a 2-cocircuit $\{a, b\}$. By Lemma 2.7, the circuit graph of $M / a$ is isomorphic to that of $M$. By induction hypothesis, the result holds.

Thus the theorem follows by induction.

Theorem 3.5. [20] Suppose that $G=G_{C}(M)$ is the circuit graph of a connected matroid $M$ and $C_{1}$ and $C_{2}$ are distinct vertices of $G$. Then $C_{1}$ and $C_{2}$ are connected by $d=\min \left\{d\left(C_{1}\right), d\left(C_{2}\right)\right\}$ edge -disjoint paths where $d\left(C_{1}\right)$ and $d\left(C_{2}\right)$ denote the degree of vertices $C_{1}$ and $C_{2}$ in $G$, respectively.

Proof. We shall prove the theorem by induction on $|E|$. When $|E|=3$, each element in $M$ is parallel to another. It is easy to see that $G=K_{3}$. The theorem is clearly true. Suppose that the result is true for $|E|=n-1$. We prove that the result is also true for $|E|=n>3$. Let $C_{1}$ and $C_{2}$ be any two vertices in $G$.

There are two cases to distinguish.

Case 1. $\left(C_{1} \cup C_{2}\right)=E$. It is easy to see that $C_{1}$ and $C_{2}$ are both adjacent to any circuit in $\mathscr{C}-\left\{C_{1} \cup C_{2}\right\}$ and the conclusion is obviously true.

Case 2. $\left(C_{1} \cup C_{2}\right) \neq E$.

There are two subcases to distinguish.

Subcase 2.1. There is an element $e \in E-\left(C_{1} \cup C_{2}\right)$ such that $M \backslash e$ is connected. Let $G_{1}=$ $G(M \backslash e)$ be the circuit graph of $M \backslash e$ and $G_{2}$ be the subgraph of $G$ induced by $V_{1}$ where $V_{1}=$ $\{C \mid C \in \mathscr{C}, e \in C\}$. Thus $C_{1}$ and $C_{2}$ are in $G_{1}$. By induction, in $G_{1}, C_{1}, C_{2}$ are connected by $d_{1}=\min \left\{d_{1}\left(C_{1}\right), d_{1}\left(C_{2}\right)\right\}$ edge-disjoint paths where $d_{1}\left(C_{1}\right)$ and $d_{1}\left(C_{2}\right)$ denote the degree of vertices $C_{1}$ and $C_{2}$ in $G_{1}$, respectively. Let $\mathscr{P}_{1}=\left\{P_{1}, P_{2}, \cdots, P_{d_{1}}\right\}$ be the family of shortest edge-disjoint paths connecting $C_{1}$ and $C_{2}$ in $G_{1}$. Without loss of generality, we may assume that $d_{1}\left(C_{1}\right) \geq d_{1}\left(C_{2}\right)$. There are two subcases to distinguish.

Subcase 2.1 a. $\quad d_{1}\left(C_{1}\right)=d_{1}\left(C_{2}\right)$. Thus $d_{1}=\min \left\{d_{1}\left(C_{1}\right), d_{1}\left(C_{2}\right)\right\}=d_{1}\left(C_{1}\right)=d_{1}\left(C_{2}\right)$. We assume that there are $m$ vertices $A_{1}, A_{2}, \cdots, A_{m}$ in $G_{2}$ that are adjacent to $C_{1}$ and $n$ vertices $D_{1}, D_{2}, \cdots, D_{n}$ in $G_{2}$ that are adjacent to $C_{2}$ where $m, n$ are integers. $G_{2}$ is a complete 
graph, so $A_{i}$ is adjacent to $D_{j}(i=1,2, \cdots, m ; j=1,2, \cdots, n)$. Here maybe $A_{i}=D_{j}$ for some $1 \leq i \leq m ; 1 \leq j \leq n$. Let $q=\min \{m, n\} . \quad C_{1} A_{i} D_{i} C_{2}(i=1,2, \cdots, q)$ are $q$ edge-disjoint paths in $G$. It is easy to see that $d\left(C_{1}\right)=d_{1}\left(C_{1}\right)+m, d\left(C_{2}\right)=d_{1}\left(C_{2}\right)+n$ and $d=\min \left\{d\left(C_{1}\right), d\left(C_{2}\right)\right\}=\min \left\{d_{1}\left(C_{1}\right)+m, d_{1}\left(C_{2}\right)+n\right\}=d_{1}\left(C_{1}\right)+\min \{m, n\}=d_{1}\left(C_{1}\right)+q$. $\mathscr{P}=\mathscr{P}_{1} \cup\left\{C_{1} A_{1} D_{1} C_{2}, C_{1} A_{2} D_{2} C_{2}, \cdots, C_{1} A_{q} D_{q} C_{2}\right\}$ are $d$ edge-disjoint paths connecting $C_{1}$ and $C_{2}$ in $G$.

Subcase 2.1 b. $d_{1}\left(C_{1}\right)>d_{1}\left(C_{2}\right)$. By induction, in $G_{1}$ there are $d_{1}=\min \left\{d_{1}\left(C_{1}\right), d_{1}\left(C_{2}\right)\right\}=$ $d_{1}\left(C_{2}\right)$ edge -disjoint paths connecting $C_{1}$ and $C_{2}$. Let $\mathscr{P}_{1}=\left\{P_{1}, P_{2}, \cdots, P_{d_{1}\left(C_{2}\right)}\right\}$ be the family of shortest edge-disjoint paths connecting $C_{1}$ and $C_{2}$ in $G_{1}$. It is obvious that each $P_{i}(i=$ $\left.1,2, \cdots, d_{1}\left(C_{2}\right)\right)$ contains exactly one vertex adjacent to $C_{1}$ and one vertex adjacent to $C_{2}$. Let $A_{1}, A_{2}, \cdots, A_{d_{1}\left(C_{1}\right)-d_{1}\left(C_{2}\right)}$ be the vertices in $G_{1}$ that are adjacent to $C_{1}$ but not contained in $d_{1}$ edge-disjoint paths. By Lemma 2.1, for any element $e^{\prime}$ in $A_{i}\left(i=1,2, \cdots, d_{1}\left(C_{1}\right)-d_{1}\left(C_{2}\right)\right)$ there is a circuit $A_{i}^{\prime}$ in $G_{2}$ containing $e$ and $e^{\prime}$, thus $A_{i} A_{i}^{\prime}$ is an edge in $G(M)$. Let $D_{1}, D_{2}, \cdots, D_{m}$ denote the vertices in $G_{2}$ that is adjacent to $C_{2}$. $G_{2}$ is a complete graph, so $A_{i}^{\prime}$ is adjacent to $D_{j}\left(i=1,2, \cdots, d_{1}\left(C_{1}\right)-d_{1}\left(C_{2}\right) ; j=1,2, \cdots, m\right)$. If $m \leq d_{1}\left(C_{1}\right)-d_{1}\left(C_{2}\right), C_{1} A_{i} A_{i}^{\prime} D_{i} C_{2}$ are $m$ edge-disjoint paths connecting $C_{1}$ and $C_{2}$ where $A_{i}^{\prime}$ can be $D_{i}(i=1,2, \cdots, m)$. Here it is possible that $A_{i}^{\prime}=A_{j}^{\prime}\left(i \neq j ; i, j=1,2, \cdots, d_{1}\left(C_{1}\right)-d_{1}\left(C_{2}\right)\right)$. But it is forbidden that $D_{i}=D_{j}(i \neq j ; i, j=1,2, \cdots, m) . d\left(C_{2}\right)=d_{1}\left(C_{2}\right)+m \leq d_{1}\left(C_{1}\right)<d\left(C_{1}\right)$, thus $d=$ $\min \left\{d\left(C_{1}\right), d\left(C_{2}\right)\right\}=d\left(C_{2}\right) . \mathscr{P}=\mathscr{P}_{1} \cup\left\{C_{1} A_{1} A_{1}^{\prime} D_{1} C_{2}, C_{1} A_{2} A_{2}^{\prime} D_{2} C_{2}, \cdots, C_{1} A_{m} A_{m}^{\prime} D_{m} C_{2}\right\}$ are $d$ edge-disjoint paths connecting $C_{1}$ and $C_{2}$ in $G$. If $m>d_{1}\left(C_{1}\right)-d_{1}\left(C_{2}\right)$, let $\mathscr{P}_{2}=\left\{C_{1} A_{1} A_{1}^{\prime} D_{1} C_{2}, C_{1} A_{2} A_{2}^{\prime} D_{2} C_{2}, \cdots, C_{1} A_{d_{1}\left(C_{1}\right)-d_{1}\left(C_{2}\right)} A_{d_{1}\left(C_{1}\right)-d_{1}\left(C_{2}\right)}^{\prime} D_{d_{1}\left(C_{1}\right)-d_{1}\left(C_{2}\right)} C_{2}\right\}$ be $d_{1}\left(C_{1}\right)-d_{1}\left(C_{2}\right)$ edge-disjoint paths connecting $C_{1}$ and $C_{2}$ where $A_{i}^{\prime}$ can be $D_{i}(i=$ $\left.1,2, \cdots, d_{1}\left(C_{1}\right)-d_{1}\left(C_{2}\right)\right)$. Let $L_{1}, L_{2}, \cdots, L_{n}$ denote the vertices in $G_{2}$ that is adjacent to $C_{1} \cdot G_{2}$ is a complete graph, so $L_{i}$ is adjacent to $D_{j}\left(i=1,2, \cdots, n ; j=d_{1}\left(C_{1}\right)-d_{1}\left(C_{2}\right)+1, d_{1}\left(C_{1}\right)-\right.$ $\left.d_{1}\left(C_{2}\right)+2, \cdots, m\right)$. If $m>n+d_{1}\left(C_{1}\right)-d_{1}\left(C_{2}\right), d\left(C_{1}\right)=d_{1}\left(C_{1}\right)+n \leq d_{1}\left(C_{2}\right)+m<d\left(C_{2}\right)$, thus $d=\min \left\{d\left(C_{1}\right), d\left(C_{2}\right)\right\}=d\left(C_{1}\right) . \mathscr{P}_{3}=C_{1} L_{i} D_{d_{1}}\left(C_{1}\right)-d_{1}\left(C_{2}\right)+i C_{2}$ are $n$ edge-disjoint paths connecting $C_{1}$ and $C_{2}$ where $L_{i}$ can be $D_{d_{1}\left(C_{1}\right)-d_{1}\left(C_{2}\right)+i}(i=1,2, \cdots, n)$. Thus $\mathscr{P}=$ $\mathscr{P}_{1} \cup \mathscr{P}_{2} \cup \mathscr{P}_{3}$ are $d=d\left(C_{1}\right)$ edge-disjoint paths in $G$. If $d_{1}\left(C_{1}\right)-d_{1}\left(C_{2}\right)<m \leq n+$ $d_{1}\left(C_{1}\right)-d_{1}\left(C_{2}\right), \mathscr{P}_{3}^{\prime}=C_{1} L_{i} D_{d_{1}\left(C_{1}\right)-d_{1}\left(C_{2}\right)+i} C_{2}$ are $m-\left(d_{1}\left(C_{1}\right)-d_{1}\left(C_{2}\right)\right.$ edge-disjoint paths connecting $C_{1}$ and $C_{2}$ where $L_{i}$ can be $D_{d_{1}\left(C_{1}\right)-d_{1}\left(C_{2}\right)+i}\left(i=1,2, \cdots, m-\left(d_{1}\left(C_{1}\right)-d_{1}\left(C_{2}\right)\right)\right.$ but $D_{d_{1}\left(C_{1}\right)-d_{1}\left(C_{2}\right)+i} \neq D_{d_{1}\left(C_{1}\right)-d_{1}\left(C_{2}\right)+j}\left(i \neq j ; i, j=1,2, \cdots, m-\left(d_{1}\left(C_{1}\right)-d_{1}\left(C_{2}\right)\right) . d\left(C_{2}\right)=\right.$ $d_{1}\left(C_{2}\right)+m \leq d_{1}\left(C_{1}\right)+n=d\left(C_{1}\right)$, thus $d=\min \left\{d\left(C_{1}\right), d\left(C_{2}\right)\right\}=d\left(C_{2}\right) . \mathscr{P}=\mathscr{P}_{1} \cup \mathscr{P}_{2} \cup \mathscr{P}_{3}^{\prime}$ are $d=d\left(C_{2}\right)$ edge-disjoint paths connecting $C_{1}$ and $C_{2}$ in $G$. The conclusion holds.

Subcase 2.2. There is no element $e \in E-\left(C_{1} \cup C_{2}\right)$ such that $M \backslash e$ is connected. If $E-\left(C_{1} \cup\right.$ $\left.C_{2}\right)=\{e\}$ and $M e$ is disconnected, it is easy to see that $C_{1} \cap C_{2}=\varnothing$ and $C_{1}, C_{2}$ are the two components of $M \backslash e$. Thus any circuit of $M$ intersecting both $C_{1}$ and $C_{2}$ contains $e$. Then $C_{1}$ and $C_{2}$ are both adjacent to any circuit in $\mathscr{C}-\left\{C_{1} \cup C_{2}\right\}$ and the conclusion is obviously true. Suppose that $\left|E-\left(C_{1} \cup C_{2}\right)\right| \geq 2$ and for any $e \in E-\left(C_{1} \cup C_{2}\right), M \backslash e$ is disconnected. By Lemma 2.5, $M$ has a 2-cocircuit $\{a, b\}$. By Lemma 2.7, the circuit graph of $M / a$ is isomorphic to that of $M$. By induction hypothesis, the result holds. Thus the theorem follows by induction.

By Theorem 3.5, we can get the following corollary.

Corollary 3.6. Suppose that $G=G(M)$ is the circuit graph of a connected matroid $M$ with minimum degree $\delta(G)$. Then the edge connectivity $\kappa^{\prime}(G)=\delta(G)$. 
Proof. By Theorem 3.5, we know that $\kappa^{\prime}(G) \geq \delta(G)$. Since for any graph $G$, we have $\kappa^{\prime}(G) \leq$ $\delta(G)$, then $\kappa^{\prime}(G)=\delta(G)$.

Theorem 3.7. [20] Let $G$ be the circuit graph of a connected matroid $M=(E, \mathscr{C})$. If $|V(G)|=n$ and $k_{1}+k_{2}+\cdots+k_{p}=n$ where $k_{i}$ is an integer, $i=1,2, \ldots, p$, then there is a partition of $V(G)$ into $p$ parts $V_{1}, V_{2}, \ldots, V_{p}$ such that $\left|V_{i}\right|=k_{i}$ and the subgraph $H_{i}$ induced by $V_{i}$ contains a $k_{i}$-cycle when $k_{i} \geq 3, H_{i}$ is isomorphic to $K_{2}$ when $k_{i}=2, H_{i}$ is a single vertex when $k_{i}=1$.

Proof. We shall prove the theorem by induction on $|E|$. When $|E|=3$ and $|V(G)|=1$, the result holds clearly. When $|E|=3$ and $|V(G)|=3, M=U_{1,3}[1]$. It is easy to see that $G=K_{3}$. The theorem is clearly true. Suppose that the result is true for $|E|=m-1$. We prove that the result is also true for $|E|=m>3$.

Case 1. There is an element $e$ in $M$ such that $M \backslash e$ is connected. Let $G_{1}$ and $G_{2}$ be the graphs defined as above. We assume that $\left|V\left(G_{1}\right)\right|=n_{1}$ and $\left|V\left(G_{2}\right)\right|=n_{2}$. There exists an $q$ such that $k_{1}+k_{2}+\cdots+k_{q-1}<n_{1}$ and $k_{1}+k_{2}+\cdots+k_{q} \geq n_{1}$. By induction, the vertices of $G(M \backslash e)$ can be partitioned into $q$ parts $V_{1}, V_{2}, \ldots, V_{q}^{\prime}$ such that $\left|V_{1}\right|=k_{1},\left|V_{2}\right|=$ $k_{2}, \ldots,\left|V_{q-1}\right|=k_{q-1},\left|V_{q}^{\prime}\right|=n_{1}-\left(k_{1}+k_{2}+\cdots+k_{q-1}\right)=k_{q}^{\prime}$ and the subgraph $H_{i}\left(H_{q}^{\prime}\right)$ induced by $V_{i}(i=1,2, \ldots, q-1)\left(V_{q}^{\prime}\right)$ contains a $k_{i}\left(k_{q}^{\prime}\right)$-cycle when $k_{i} \geq 3\left(k_{q}^{\prime} \geq 3\right), H_{i}\left(H_{q}^{\prime}\right)$ is isomorphic to $K_{2}$ when $k_{i}=2\left(k_{q}^{\prime}=2\right), H_{i}\left(H_{q}^{\prime}\right)$ is a single point when $k_{i}=1\left(k_{q}^{\prime}=1\right)$. When $k_{q}=k_{q}^{\prime}$, the result holds clearly because $G_{2}$ is a complete graph. When $k_{q}>k_{q}^{\prime}$, there are three subcases to consider.

Subcase 1.1. $\quad k_{q}^{\prime}=1$. Suppose that $C_{1}$ is the subgraph in $G(M \backslash e)$ induced by $V_{q}^{\prime}$. When $k_{q}=2$, obviously there is a vertex in $G_{2}$ that is adjacent to $C_{1}$. When $k_{q} \geq 3$, we prove that there is a 3-cycle $C_{1} C_{2} C_{3}$ in $G$ such that $C_{2} C_{3} \in E\left(G_{2}\right)$. For any $e_{1} \in C_{1}$, by Lemma 2.1, there is a circuit $C_{2} \in G_{2}$ containing $e_{1}$ and $e$. Let $e_{2} \in C_{1}-C_{2}$. There is a circuit $C_{3} \in G_{2}$ containing $e_{2}$ and $e$. We get the 3-cycle $C_{1} C_{2} C_{3}$. Note that $G_{2}$ is a complete graph. In $G_{2}$ there is a $k_{q}-2$ path $P$ connecting $C_{2}$ and $C_{3} . C_{1} C_{2}+P+C_{3} C_{1}$ is a $k_{q}$-cycle in $G$. Because $G_{2}$ is a complete graph, the subgraph induced by $V\left(G_{2}\right)-V(P)$ is also a complete graph. Thus the vertices of the subgraph induced by $V\left(G_{2}\right)-V(P)$ can be partitioned into $p-q$ parts $V_{q+1}, V_{q+2}, \ldots, V_{p}$ such that $\left|V_{i}\right|=k_{i}, i=q+1, q+2, \ldots, p$, and the subgraph $H_{i}$ induced by $V_{i}$ contains a $k_{i}$ -cycle when $k_{i} \geq 3, H_{i}$ is isomorphic to $K_{2}$ when $k_{i}=2, H_{i}$ is a single point when $k_{i}=1$. The result holds.

Subcase 1.2. $\quad k_{q}^{\prime}=2$. Suppose that $C_{1} C_{2}$ is the subgraph in $G(M \backslash e)$ induced by $V_{q}^{\prime}$. When $k_{q}=3$, by Lemma 2.8, the result holds. When $k_{q} \geq 4$, by Lemma 2.8, there is a 4-cycle $C_{1} C_{2} C_{3} C_{4}$ in $G$ such that $C_{3} \in V\left(G_{2}\right)$ and $C_{4} \in V\left(G_{2}\right)$ and $C_{1} C_{2} C_{3}$ is a 3-cycle of $G$. In $G_{2}$ there is a $k_{q}-3$-path $P$ connecting $C_{3}$ and $C_{4} . C_{1} C_{2}+C_{2} C_{3}+P+C_{4} C_{1}$ is a $k_{q}$-cycle in $G$. Because $G_{2}$ is a complete graph, the subgraph induced by $V\left(G_{2}\right)-V(P)$ is also a complete graph. Thus the result holds.

Subcase 1.3. $\quad k_{q}^{\prime}>2$. The subgraph $H_{q}^{\prime}$ in $G(M \backslash e)$ induced by $V_{q}^{\prime}$ contains a $k_{q}^{\prime}$-cycle. Let $C_{1} C_{2}$ be any edge in this cycle and $P_{1}$ be the Hamilton path in $H_{q}^{\prime}$ connecting $C_{1}$ and $C_{2}$. By Lemma 2.8, there is a 4-cycle $C_{1} C_{2} C_{3} C_{4}$ in $G$ such that $C_{3} C_{4} \in E\left(G_{2}\right)$ and $C_{1} C_{2} C_{3}$ is a 3-cycle of $G$. When $k_{q}-k_{q}^{\prime}=1, P_{1}+C_{2} C_{3}+C_{3} C_{1}$ is a $k_{q}$-cycle in $G$. When $k_{q}-k_{q}^{\prime} \geq 2$, in $G_{2}$ there is a $k_{q}-k_{q}^{\prime}-1$-path $P_{2}$ connecting $C_{3}$ and $C_{4} . P_{1}+C_{2} C_{3}+P_{2}+C_{4} C_{1}$ is a $k_{q}$-cycle in $G$. Note that 
$G_{2}$ is a complete graph. The subgraph induced by $V\left(G_{2}\right)-V\left(P_{2}\right)$ is also a complete graph. Thus the result holds.

Case 2. The matroid $M$ is critically connected. By Lemma 2.2 , for any element $e$ in $M, M / e$ is connected. By Lemma 2.5, $M$ has a 2-cocircuit $\{a, b\}$. By Lemma 2.7, the circuit graph of $M / a$ is isomorphic to that of $M$. By induction hypothesis, the result holds.

Thus the theorem follows by induction.

From Theorem 3.7 we have the following theorem holds.

Theorem 3.8. Let $G=G(M)$ be the circuit graph of a connected matroid $M=(E, \mathcal{C})$. If $|V(G)|=n$ and $k_{1}+k_{2}+\cdots+k_{p}=n$ where $k_{i}$ is an integer and $k_{i} \geq 3, i=1,2, \ldots, p$, then $G$ has a 2-factor $F$ containing $p$ vertex-disjoint cycles $D_{1}, D_{2}, \ldots, D_{p}$ such that the length of $D_{i}$ is $k_{i}(i=1,2, \ldots, p)$.

By the similar methods we can prove that the above theorems also holds for the intersection graph of bases of matroids.

Finally, We present the following open problems to be considered.

Problem 1. Let $G=G(M)$ be the circuit graph of a connected matroid $M=(E, \mathcal{C})$. If $|V(G)|=n$ and $C_{1}, C_{2} \in V(G)$ with $d\left(C_{1}, C_{2}\right)=r$, how many Hamilton paths connect $C_{1}$ and $C_{2}$ in $G$ ? Furthermore, how many $k$-paths connect $C_{1}$ and $C_{2}$ in $G(r \leq k \leq n-1)$ ?

Problem 2. Let $G=G(M)$ be the intersection graph of bases of matroid $M=(E, \mathcal{B})$. If $|V(G)|=n$ and $B_{1}, B_{2} \in V(G)$ with $d\left(B_{1}, B_{2}\right)=r$, how many Hamilton paths connect $B_{1}$ and $B_{2}$ in $G$ ? Furthermore, how many $k$-paths connect $B_{1}$ and $B_{2}$ in $G(r \leq k \leq n-1)$ ?

Other related results of graphs on matroids can be found in [22-60].

\section{References}

[1] J. G. Oxley, Matroid theroy, Oxford University Press, New York, 1992.

[2] J. A. Bondy, U. S. R. Murty, Graph Theory With Applications, American Elsevier, New York, 1976.

[3] S. B. Maurer, Matroid basis graphs I, J.Comb. Theory B, 14 (1973), 216-240.

[4] S. B. Maurer, Matroid basis graphs II, J. Comb. Theory B, 15 (1973) 121-145.

[5] R. L. Cummins, Hamilton circuits in tree graphs. IEEE Trans Circuit theory, 1 (1966), 82-90.

[6] C. A. Holzmann, P. G. Norton and M. D. Tobey. A graphical representation of matroids. SIAM J Appl Math., 29 (1973), 618-672.

[7] B. Alspach and G. Liu. Paths and cycles in matroid base graphs, Graphs and combinatorics, 1989, 5(3), 207-211.

[8] G. Liu. The connectivities of matroid base graphs, J. Operations Research, 3(1) (1984), 67-68.

[9] G. Liu, Matroids complexes-geometrical representations on matroids. Acta Math. Scientia, 5 (1985), 35-42. 
[10] G. Liu, The Connectivities of Adjacent Tree Graphs. Acta Mathematicae Applicatae Sinica, 3(4) (1987), 313-317.

[11] G. Liu. A lower bound on connectivities of matroid base graphs, Discrete Math., 69(1) (1988), 55-60.

[12] G. Liu. On connectivities of base graph of some matroids, J. Sys. Sci. and Math. Scis., 1(1) (1988), 18-21.

[13] G. Liu, On connectivities of tree graphs, J. Graph Theory, 12(3) (1988), 453-459.

[14] G. Liu, The proof of a conjecture on matroid basis graphs. Scince Sinica, 1990: 593-599.

[15] Liu Guizhen and Chen Qinghua, Matroids, Publishing House of National University Defense Technology, Changsha, (in chinese) 1994.

[16] Guizhen Liu and L. Zhang, Forest graphs of graphs, Chinese Journal of Engineering Mathmatics, 22 (6) (2005), 1100-1109.

[17] P. Li, Guizhen Liu, Cycles in circuit graphs of matroids, Graphs and Combinatorics 23 (2007), 425-431.

[18] Yinghao Zhang and Guizhen Liu, On Properties of the intersection graphs of matroids, to appear in Frontiers of Mathematics.

[19] H. Deng and F. Xia. The $P_{3}$-Hamiltonian property of matroid base graphs. Jour Nat Sci Hunan Norm Uni., 1 (2000), 5-8.

[20] D. Deng and R. Li. The 1-Hamiltonian property of matroid base graphs. Acta. Scinat Univ. Norm. Hunan., 22 (3) (1999), 1-5.

[21] L. Li, Matroids and Graphs, Ph.D. Dissertation, Shandong University, (2005).

[22] Ping Li, Guizhen Liu , Hamilton cycle in circuit graphs of matroids, Computer and mathematics with Applications 55 (2008), 654-659

[23] Ping Li, Some Properties of Circuit Graphs of Matroids, Doctoral Dissertation, Shandong University, (2010)

[24] B. Bollobas, A lower bound for the number of nonisomorphic Matroids, J. Comb. Theory, 1969, 7, 366-368.

[25] J. A. Bondy, Transversal matroids, base orderable matroids and graphs, Quart. J. Math., 1972, 23, 81-89.

[26] J. A. Bondy, A.W. Ingleton, Pancyclic graph II, J.Comb. Theory, B20,1976, 41-46

[27] R. A. Brualdi, On foundamental transversal matroids, Proc. Amer. Math. Soc., 45 (1974), 151-156.

[28] R. A. Brualdi, G. W. Dinolt, Characterization of transversal matroids and their presentations, J. Comb. Theory, 12 (1972), 268-286.

[29] M. Cai, A solution of Chartand's problem on spanning trees, Acta Mathematicae Applicatae Sinica (English Ser.), 1:2 (1984), 97-98.

[30] P. A. Catlin, J. W. Grossman, A. M. Hobbs, and H. J. Lai. Fractional arboricity, strength, and principal partitions in graphs and matroids. Disc. Appl. Math., 40(1992), 285-302.

[31] H. H. Crapo, Single element extensions of Matroids, J. Res. Nat. Bur. Stand., 69B (1965), 57-65.

[32] J. Donald, C. Holzmann and M. Tobey. A characterization of complete matroid base graphs, J. Comb. Theory Ser., 22B (1977), 139-193.

[33] J. R. Edmonds, Minimum partition of a matroid into indedent subsets. J.Res. Natl. Bur. Stand., 69B(1965), 67-72. 
[34] J. R. Edmonds, Matroids and the greedy algorithm, Math Programming, 1, (1971), 127-136.

[35] V. Estivill-Castro, M. Noy and J. Urrutia, On the chromatic number of tree graphs. Discrete Math., 223 (2000), 363-366.

[36] J. Gao, Quasi-1-Hamilton connectedness of tree graphs. Math. Res. and Exposition, 7(3) (1987), 498.

[37] J. Gao, 1-Hamilton connectedness of tree graphs. Mathematica Applicata., 6(2) (1993), 136-144.

[38] H. Harary, R. J. Mokken and M. J. Plantholt, Interpolation theorem for diameters of spanning trees. IEEE Trans Circuits and System, 30 (1983), 429-432.

[39] F. Harary and M. J. Plantholt, Classification of interpolation theorems for spanning trees and other families of spanning subgraphs. J. Graph Theory, 13(6)(1989), 703-712.

[40] R. Rado, A theorem on independence relations. Quart. J. Math. Oxford Ser., 13 (1942), 83-89.

[41] C. A. Holzmann, F. Harary, On the tree graph of a matroid. SIAM J. Appl. Math., 22 (1972), 187-193.

[42] A. W. Ingleton, Gammoids and transversal matroids, J. Comb. Theory, 15 (1973), 51-68.

[43] T. Kamae. The existence of Hamilton circuit in a tree graph. IEEE Trans Circuit theory, 14 (1967), 279-283.

[44] G. Kishi and Y. Kajitani. On Hamilton circuits in a tree graphs. IEEE Trans Circuit theory, 15 (1968), 42-50.

[45] E. Lawler. Combinatorial Optimization. John Wiley, New York, 1972.

[46] L. Li, The Hamilton properties of tree graphs. J. Shandong Unv. of Technology, 27 (3) (1997), 261-263.

[47] L. Li and G. Liu, The connectivities of the adjacency leaf exchange forest graphs, J. Shandong University, 39 (6) (2004), 49-51.(in Chinese)

[48] L. Li, Q. Bian and G. Liu, The matroid incidence graphs, J. Shandong University, 40 (2) (2005), 24-40.(in Chinese)

[49] L. Li, Matroids and Graphs, Ph.D. Dissertation, Shandong University, (2005).

[50] X. Li. The connectivities of the SEE-graph and the $A E E$-graph for the connected spanning $k$-edge subgraphs of a graph Discete Math., 183 (1998), 237-245.

[51] X. Li, V. Neumann-Lara and E. Rivera-Campo, Two Approaches for the Generalization of Leaf Edge Exchange Graphs on Spanning Trees to Connected Spanning k-Edge Subgraphs of a Graph. Ars. combin., 75 (2005), 257-265.

[52] L. Lovasz, A. Recski, On the sum of Matroids, Acta Math. Acad. Sci. Hung, 24 (1973), 329-333.

[53] S. B. Maurer, Intervals in matroid basis graphs. Discrete Math., 11 (1975), 147-159.

[54] U. S. R. Murty, On the number of bases of matroid, Proc. Second Louisiana Conference on Combinatorics, (1971), 387-410.

[55] U.S.R. Murty, Extremal critically connected matroids, Discrete Math., 8 (1974), 49-58.

[56] D. Naddef and W. R. Pulleyblank. Hamiltonicity and combinatorial polyhedra. J. Combin. theory B, 31 (1981), 279-312.

[57] C. St. J. A. Nash-Williams, Edge-disjiont spanning trees of finite graphs. J. London Math. Soc., 36 (1961), 445-450. 
[58] C. St. J. A. Nash-Williams, Decompositions of finite graphs. J. London Math. Soc., 39 (1964), 12.

[59] J. G. Oxley, Matroid Theory, Oxford University Press, New York, 1992.

[60] M. J. Piff, An upper bound for the number of matroids. J. Comb. Theory, 13 (1973), 241-245. 


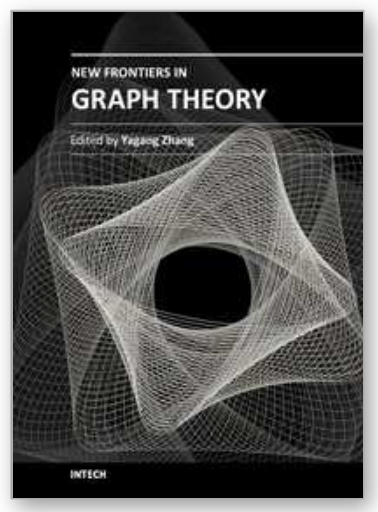

\author{
New Frontiers in Graph Theory \\ Edited by Dr. Yagang Zhang
}

ISBN 978-953-51-0115-4

Hard cover, 526 pages

Publisher InTech

Published online 02, March, 2012

Published in print edition March, 2012

Nowadays, graph theory is an important analysis tool in mathematics and computer science. Because of the inherent simplicity of graph theory, it can be used to model many different physical and abstract systems such as transportation and communication networks, models for business administration, political science, and psychology and so on. The purpose of this book is not only to present the latest state and development tendencies of graph theory, but to bring the reader far enough along the way to enable him to embark on the research problems of his own. Taking into account the large amount of knowledge about graph theory and practice presented in the book, it has two major parts: theoretical researches and applications. The book is also intended for both graduate and postgraduate students in fields such as mathematics, computer science, system sciences, biology, engineering, cybernetics, and social sciences, and as a reference for software professionals and practitioners.

\title{
How to reference
}

In order to correctly reference this scholarly work, feel free to copy and paste the following:

Ping Li and Guizhen Liu (2012). The Properties of Graphs of Matroids, New Frontiers in Graph Theory, Dr. Yagang Zhang (Ed.), ISBN: 978-953-51-0115-4, InTech, Available from:

http://www.intechopen.com/books/new-frontiers-in-graph-theory/the-properties-of-graphs-of-matroids

\section{INTECH}

open science | open minds

\author{
InTech Europe \\ University Campus STeP Ri \\ Slavka Krautzeka 83/A \\ 51000 Rijeka, Croatia \\ Phone: +385 (51) 770447 \\ Fax: +385 (51) 686166 \\ www.intechopen.com
}

\author{
InTech China \\ Unit 405, Office Block, Hotel Equatorial Shanghai \\ No.65, Yan An Road (West), Shanghai, 200040, China \\ 中国上海市延安西路65号上海国际贵都大饭店办公楼 405 单元 \\ Phone: +86-21-62489820 \\ Fax: +86-21-62489821
}


(C) 2012 The Author(s). Licensee IntechOpen. This is an open access article distributed under the terms of the Creative Commons Attribution 3.0 License, which permits unrestricted use, distribution, and reproduction in any medium, provided the original work is properly cited. 